\title{
Implementation of overlapping soliton pair to reduce time delay in femtosecond pulse propagation over short standard single mode fiber
}

\begin{abstract}
Propagation of 50 femtosecond soliton pulse over short Standard Single Mode Fiber (SSMF) is reported by using soliton pair as the input pulse. The attraction of two solitons to propagate as one pulse is intended. The time deviation and average amplitude of propagating pulses are compared for several soliton orders and initial inter-pulse delays. It is discovered that by considering overlapping soliton pair as the input pulse, under special conditions, better propagation characteristic and lower time delay is experienced over short SSMF in comparison to the fundamental soliton.
\end{abstract}

Keyword: Delay reduction; Femtosecond soliton; Nonlinear Schrödinger equation; Overlapping soliton pair 